\title{
Generation of an Annual Typical Meteorological Solar Irradiance on Tilted Surfaces for Armidale NSW,Australia
}

\author{
Yasser Maklad ${ }^{1}$ \\ ${ }^{1}$ (University of New England, Armidale NSW 2351 NSW Australia-School of Environmental \& Rural Science - \\ email: ymaklad@myune.edu.au)
}

\begin{abstract}
This paper aims to estimate solar irradiance on tilted surfaces for Armidale NSW, Australia.The most common data for describing the local solar climate is through what is called Typical Meteorological Year data (TMY). Typical solar radiation data is very important for the calculations of many solarapplications. In this study, typical solar radiation years on tilted surfaces of five inclination angles $\left(15^{\circ}, 30^{\circ}, 45^{\circ}, 60^{\circ}\right.$ and $\left.75^{\circ}\right)$ for Armidale town in New South Wales in Australia are generated from the typical solar radiation years on horizontal surfaces developed earlier by the writer in a former article based on the daily and monthly solar radiation data measured and collected for 23 years during the period 1990 to 2012, utilising the FinkelsteinSchafer statistical method. The study outcome is expected to show how solar radiation is potential in Armidale NSW and would be a real help for solar energy generation systems' designers in this region for all building applications varying between residential, educational, administrative and commercial for sizing and maximising efficiency of such systems by using the tabular TYR outcome for the each day of the year. Specially for calculating the energy output of solar arrays through the day while the sun is moving around the fixed angle array surface. This would really give a real picture of actual energy generation utilising inclined solar arrays.
\end{abstract}

Keywords: Armidale NSW,test meteorological year, test reference year, solar radiation; tilted surfaces

\section{INTRODUCTION}

The most common data for describing the local solarclimate is through what is called Typical Meteorological Year data (TMY). To determine TMY data, various meteorological measurements are made at hourly intervals over a number of years to build up a picture of the local climate. A simple average of the yearly data underestimates the amount of variability, so the month that is most representative of the location is selected. For each month, the average solar radiation over the whole measurement period is determined, together with the average solar radiation in each month during the measurement period. The data for the month that has the average solar radiation most closely equal to the monthly average over the whole measurement period is then chosen as the TMY data for that month. This process is then repeated for each month in the year. The months are added together to give a full year of hourly samples. There is no strict standard for TMY data so the user must adjust the data to suit the application. Considerable care must be taken with sample periods. Solar radiation data is a crucial parameter for the prediction of long-term performance of solar energy generation systems. As well, it is a key input in modelling and designing of solar energy applications. Thus, a need for a reliable source of solar radiationdata has to be readily available for particular settlement locations.

The need for a one-year representative daily meteorological data led to the development of methodologies known as the Typical Meteorological Year (TMY), alternatively called Test Reference Year (TRY) [1]. TMY or TRY is a representative data that consists of the month selected from the individual years and concatenated to form a complete year. However, A TMY is not necessarily a good indicator of conditions over the next year or even the next five years. Rather, TMY represents conditions judged to be typical over a long period of time [2]. Typical weather year data sets can be generated for several climatic variables such as temperature, humidity, wind speed, etc. or only for solar radiation speed. Various trials have been made to generate such weather databases for different areas around the world [1, 3, 4, 5, 6, 7, 8, 9, 10, 11, 13 \& 14].

A variety of numerical models for calculating solar irradiance on tilted surfaceswere described and evaluated using data for any location on the earth. While all the hourly models have a common approach for calculating the direct component of solar irradiance, there are a variety of methods for calculating diffuse irradiance based on the portion of the sky hemisphere within the field of view of the surface. A less significant distinction between the models is in the methods used to calculate the amount of radiation received as a result of reflection from adjacent surfaces [16].

The hourly solar radiation data required for solar energy system design evaluation and performance studies are generally not available for a number of sites, especially for those in remote locations. As such, accurate determination of hourly solar radiation data is important at both horizontal surfaces and inclined surfaces. A model to estimate global solar radiation using temperature and sunshine hour data has been 
developed [17], which is used to calculate the hourly solar radiation data. The hourly solar radiation has also been calculated using [18] daily integration approach from the measured daily solar radiation data. These two predicted hourly solar radiation data values are compared with measured hourly values to test the accuracy of the models. The total solar radiation on the inclined surfaces and vertical surfaces for different orientations has also been estimated. The estimated values are found to be in close agreement with the measured values. The presented method can be used to estimate hourly, global, diffuse solar radiation for horizontal surfaces and total solar radiation on inclined and vertical surfaces at different orientations with a greater accuracy for any location.

Thus, the main aim of this study is to generate representative TYM solar radiation data on tilted surfaces for Armidale NSW, Australia. Inclination angles considered are $\left(15^{\circ}, 30^{\circ}, 45^{\circ}, 60^{\circ}\right.$ and $\left.75^{\circ}\right)$.

\section{DATA AND LOCATION}

The daily mean solar radiation recorded during the period 1994-2010 are utilized to generate the typical solar radiation data. In Australia, meteorological observations are recorded by the Australian Bureau of Meteorology (BOM) weather stations are widely spreader in lots of cities and towns around Australia. In this study, the global solar radiation data recorded by Armidale Airport Weather Automatic Station and published on the BOM's website where it was collected. The missing and invalid measurements account for approximately $0.01 \%$ of the whole database of mean solar radiation; those were replaced with the values of preceding or subsequent days by interpolation. During the calculations process, any year found with more than ten days in any month observations not available was excluded. "Table 1" provides geographical information for Armidale town and the periods of the relevant mean solar radiation data.

Table 1 Geographical and mean solar radiation database information of Armidale NSW, Australia Longitude ( $†$ E) Latitude $(† \mathrm{~S}) \quad$ Elevation (m) Daily Mean Solar Radiation

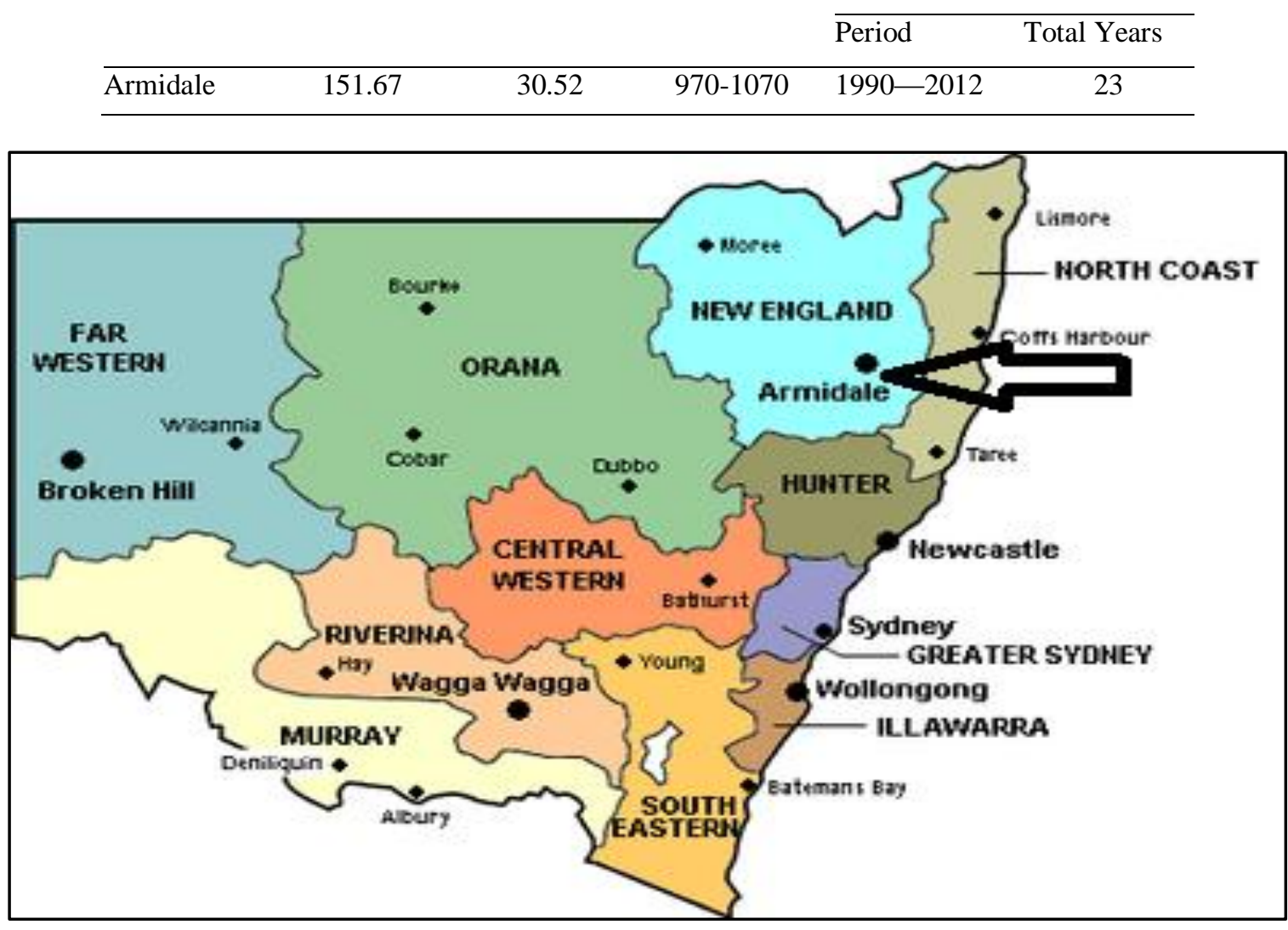

Figure 1 Armidale NSW, Australia location

\section{METHODOLOGY}

The power incident on a photo voltaic (PV) module depends not only on the power contained in the sunlight, but also on the angle between the module and the sun. When the absorbing surface and the sunlight are perpendicular to each other, the power density on the surface is equal to that of the sunlight (in other words, the power density will always be at its maximum when the PV module is perpendicular to the sun). However, as the 
angle between the sun and a fixed surface is continually changing, the power density on a fixed PV module is less than that of the incident sunlight.

The amount of solar radiation incident on a tilted module surface is the component of the incident solar radiation which is perpendicular to the module surface. The following figure shows how to calculate the radiation incident on a tilted surface $\left(\mathrm{S}_{\text {module }}\right)$ given either the solar radiation measured on horizontal surface $\left(S_{\text {horiz }}\right)$ or the solar radiation measured perpendicular to the sun $\left(S_{\text {incident }}\right)$.

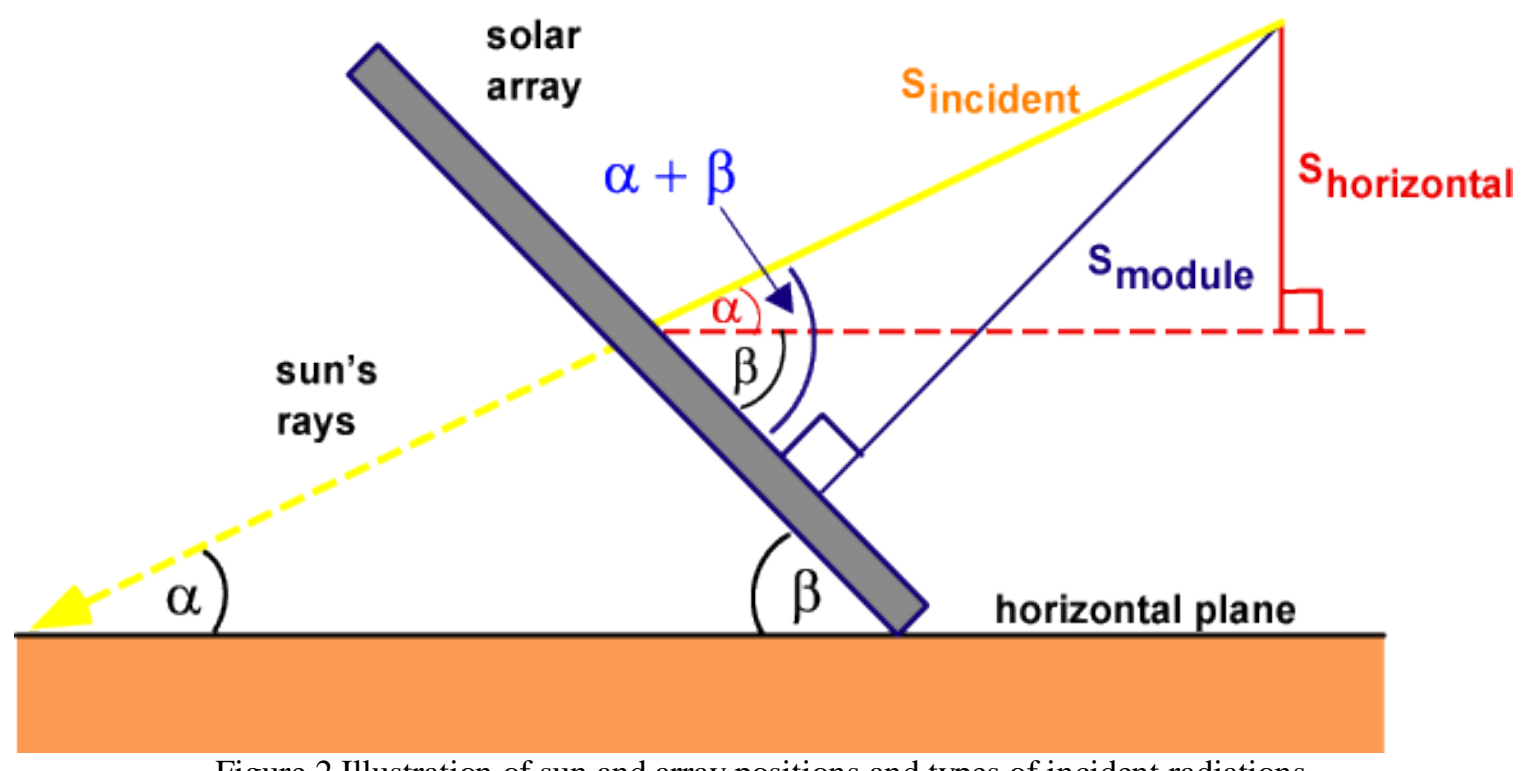

Figure 2 Illustration of sun and array positions and types of incident radiations

The equations relating $\mathrm{S}_{\text {module }}, \mathrm{S}_{\text {horiz }}$ and $\mathrm{S}_{\text {incident }}$ are:

\section{$S_{\text {horizontal }}=S_{\text {incident }} \sin \alpha$ \\ $S_{\text {module }}=S_{\text {incident }} \sin (\alpha+\beta)$}

where

$\alpha$ is the elevation angle; and

$\beta$ is the tilt angle of the module measured from the horizontal.

The elevation angle has been previously given as:

$$
\alpha=90-\phi+\delta
$$

where $\varphi$ is the latitude; and

$\delta$ is the declination angle previously given as:

$$
\delta=23.45^{\circ} \sin \left[\frac{360}{365}(284+d)\right]
$$

where $d$ is the day of the year. Note that from simple math $(284+d)$ is equivalent to (d-81) which was used before. Two equations are used interchangeably in literature.

From these equations a relationship between $S_{\text {module }}$ and $S_{\text {horiz }}$ can be determined as:

$$
S_{\text {module }}=\frac{S_{\text {horizontal }} \sin (\alpha+\beta)}{\sin \alpha}
$$

\section{GENERATION OF TYPICAL SOLAR RADIATION YEAON TILTED SURFACES}

Applying the above methodology for all the months in the database, the Test Reference Year for daily solar radiation data was formed for Armidale.

The test reference years with minimum FS for monthly mean global solar radiation for Armidale are given in "Table 2" [15]. Which shows that, although the big picture that Armidale has a high potential of solarenergy, still there are considerable differences of potentiality in through the months due to the fact that Armidale's winter season (June, July and August) is relatively cloudy. "Table 2", the minimum and maximum values of monthly mean of the daily global solar radiation on a horizontal surface (ITRY) in Armidale, the minimum is $10.41 \mathrm{MJ} / \mathrm{m} 2$ day in June and the maximum is $25.88 \mathrm{MJ} / \mathrm{m} 2$ day in December. 
Table2TestReferenceYearswithminimum (min)FSandmonthlymeanofthesolar radiation(ITRY) for Armidale NSW, Australia

\begin{tabular}{|c|c|c|}
\hline Month & Year & ITRY $\left(\mathrm{MJ} / \mathrm{m}^{2}\right.$ day $)$ \\
\hline January & 1993 & 25.33 \\
\hline February & 2007 & 25.18 \\
\hline March & 1993 & 19.68 \\
\hline April & 1993 & 15.98 \\
\hline May & 2000 & 12.4 \\
\hline June & 2006 & 10.41 \\
\hline July & 1991 & 11.44 \\
\hline August & 1999 & 14.62 \\
\hline September & 2012 & 19.11 \\
\hline October & 1991 & 22.00 \\
\hline November & 1991 & 23.59 \\
\hline December & 1994 & 25.88 \\
\hline & Extracted from $[15]$ & \\
\hline
\end{tabular}

By applying the methodology to test reference years of horizontal solar radiation data shown in table 3 , solar irradiance for module (array) and incident solar radiation are gained for each day of the reference year for prescribed inclination angles $\left(15^{\circ}, 30^{\circ}, 45^{\circ}, 60^{\circ}\right.$ and $\left.75^{\circ}\right)$ as tabulated in tables $[4,5,6,7,8,9,10,11,12,13,14$ $\& 15]$, each table represents a month with each day separately.

Table3Daily solar radiation on horizontal surfaces valuesobtainedfromTestReferenceYear data for Armidale NSW, Australia

\begin{tabular}{|c|c|c|c|c|c|c|c|c|c|c|c|c|}
\hline Day & Jan & Feb & Mar & Apr & May & Jun & $\mathrm{Jul}$ & Aug & Sep & Oct & Nov & Dec \\
\hline 1 & 29.4 & 18.1 & 19.1 & 18.8 & 13.5 & 10.8 & 10.0 & 14.5 & 16.4 & 20.5 & 22.3 & 24.6 \\
\hline 2 & 27.0 & 22.5 & 21.8 & 18.9 & 12.6 & 10.6 & 10.4 & 14.2 & 16.3 & 19.4 & 22.9 & 25.0 \\
\hline 3 & 27.2 & 23.2 & 21.1 & 17.8 & 13.9 & 9.00 & 11.2 & 13.6 & 16.1 & 19.3 & 23.4 & 26.0 \\
\hline 4 & 26.1 & 26.1 & 22.0 & 19.0 & 13.0 & 10.7 & 10.0 & 13.2 & 17.5 & 20.9 & 23.1 & 25.7 \\
\hline 5 & 23.2 & 25.5 & 19.8 & 16.8 & 12.0 & 10.9 & 12.0 & 13.3 & 15.8 & 21.2 & 21.3 & 25.4 \\
\hline 6 & 23.2 & 23.5 & 21.3 & 19.5 & 14.7 & 11.6 & 11.2 & 14.3 & 18.7 & 19.9 & 22.3 & 23.8 \\
\hline 7 & 26.6 & 21.5 & 19.4 & 18.8 & 14.5 & 9.70 & 10.8 & 13.7 & 17.0 & 21.4 & 24.1 & 26.1 \\
\hline 8 & 25.1 & 23.2 & 20.3 & 16.4 & 12.8 & 10.3 & 10.7 & 14.9 & 17.0 & 21.6 & 22.0 & 26.8 \\
\hline 9 & 24.9 & 23.2 & 19.5 & 16.2 & 13.4 & 10.6 & 9.60 & 14.9 & 15.7 & 22.3 & 23.2 & 24.8 \\
\hline 10 & 26.4 & 24.1 & 21.0 & 17.4 & 12.9 & 10.7 & 11.2 & 15.4 & 18.7 & 21.5 & 24.7 & 22.7 \\
\hline 11 & 26.4 & 24.3 & 21.4 & 16.3 & 12.8 & 10.1 & 11.4 & 15.1 & 18.3 & 22.4 & 24.2 & 24.7 \\
\hline 12 & 26.7 & 21.4 & 20.2 & 16.5 & 12.6 & 11.6 & 10.3 & 15.2 & 18.4 & 22.8 & 25.3 & 26.3 \\
\hline 13 & 26.1 & 22.4 & 21.0 & 16.4 & 12.5 & 9.80 & 9.90 & 15.7 & 20.4 & 23.7 & 24.4 & 28.3 \\
\hline 14 & 25.6 & 20.2 & 21.1 & 14.9 & 12.7 & 10.2 & 10.2 & 15.6 & 19.7 & 21.9 & 26.9 & 25.8 \\
\hline 15 & 25.7 & 19.7 & 21.9 & 16.7 & 12.9 & 10.5 & 11.6 & 15.6 & 19.3 & 23.2 & 23.8 & 25.2 \\
\hline 16 & 26.6 & 20.3 & 21.9 & 15.7 & 12.2 & 10.5 & 12.4 & 14.3 & 19.7 & 23.6 & 22.5 & 27.0 \\
\hline 17 & 25.1 & 23.9 & 19.3 & 15.4 & 11.5 & 11.2 & 12.1 & 14.4 & 19.2 & 22.5 & 21.3 & 28.3 \\
\hline 18 & 25.1 & 22.2 & 20.7 & 16.2 & 11.1 & 10.6 & 10.7 & 14.0 & 19.7 & 23.0 & 21.5 & 26.0 \\
\hline 19 & 22.1 & 21.8 & 20.9 & 15.2 & 12.4 & 9.50 & 12.2 & 14.6 & 20.0 & 21.8 & 25.5 & 27.1 \\
\hline 20 & 28.4 & 22.8 & 20.5 & 15.4 & 12.4 & 9.90 & 12.4 & 13.9 & 19.9 & 23.6 & 24.2 & 26.8 \\
\hline 21 & 27.3 & 23.4 & 19.8 & 16.2 & 11.3 & 10.6 & 12.4 & 15.5 & 19.9 & 22.9 & 21.3 & 28.1 \\
\hline 22 & 25.0 & 21.3 & 17.8 & 15.7 & 11.9 & 10.6 & 12.2 & 14.3 & 21.2 & 24.3 & 24.2 & 26.4 \\
\hline 23 & 23.5 & 23.2 & 18.8 & 16.2 & 12.2 & 10.4 & 12.7 & 12.8 & 21.8 & 24.4 & 23.7 & 27.9 \\
\hline 24 & 25.6 & 23.3 & 20.3 & 14.6 & 12.0 & 11.1 & 11.6 & 16.1 & 20.2 & 21.3 & 25.6 & 24.3 \\
\hline 25 & 20.8 & 19.0 & 19.5 & 14.6 & 12.2 & 11.0 & 10.8 & 16.8 & 21.5 & 22.9 & 25.3 & 26.2 \\
\hline 26 & 25.4 & 21.5 & 17.8 & 14.9 & 11.9 & 10.3 & 9.60 & 16.2 & 20.5 & 20.0 & 26.3 & 26.3 \\
\hline 27 & 25.2 & 21.5 & 19.4 & 13.7 & 12.2 & 9.80 & 11.4 & 15.5 & 21.5 & 21.3 & 26.1 & 19.8 \\
\hline 28 & 27.2 & 25.5 & 17.9 & 13.1 & 11.7 & 10.6 & 11.7 & 15.3 & 21.6 & 26.9 & 26.5 & 26.2 \\
\hline 29 & 26.8 & 18.3 & 18.5 & 15.1 & 11.4 & 11.2 & 13.6 & 14.9 & 22.1 & 25.5 & 25.7 & 28.3 \\
\hline 30 & 27.4 & & 15.8 & 14.9 & 11.5 & 9.70 & 13.3 & 14.0 & 23.7 & 21.3 & 26.0 & 26.1 \\
\hline 31 & 22.8 & & 18.7 & & 11.0 & & 12.8 & 15.9 & & 22.6 & & 28.4 \\
\hline
\end{tabular}

Extracted from [15] 
Table 4 January's Daily solar radiation on tilted surfaces valuesobtainedfromTestReferenceYear data for

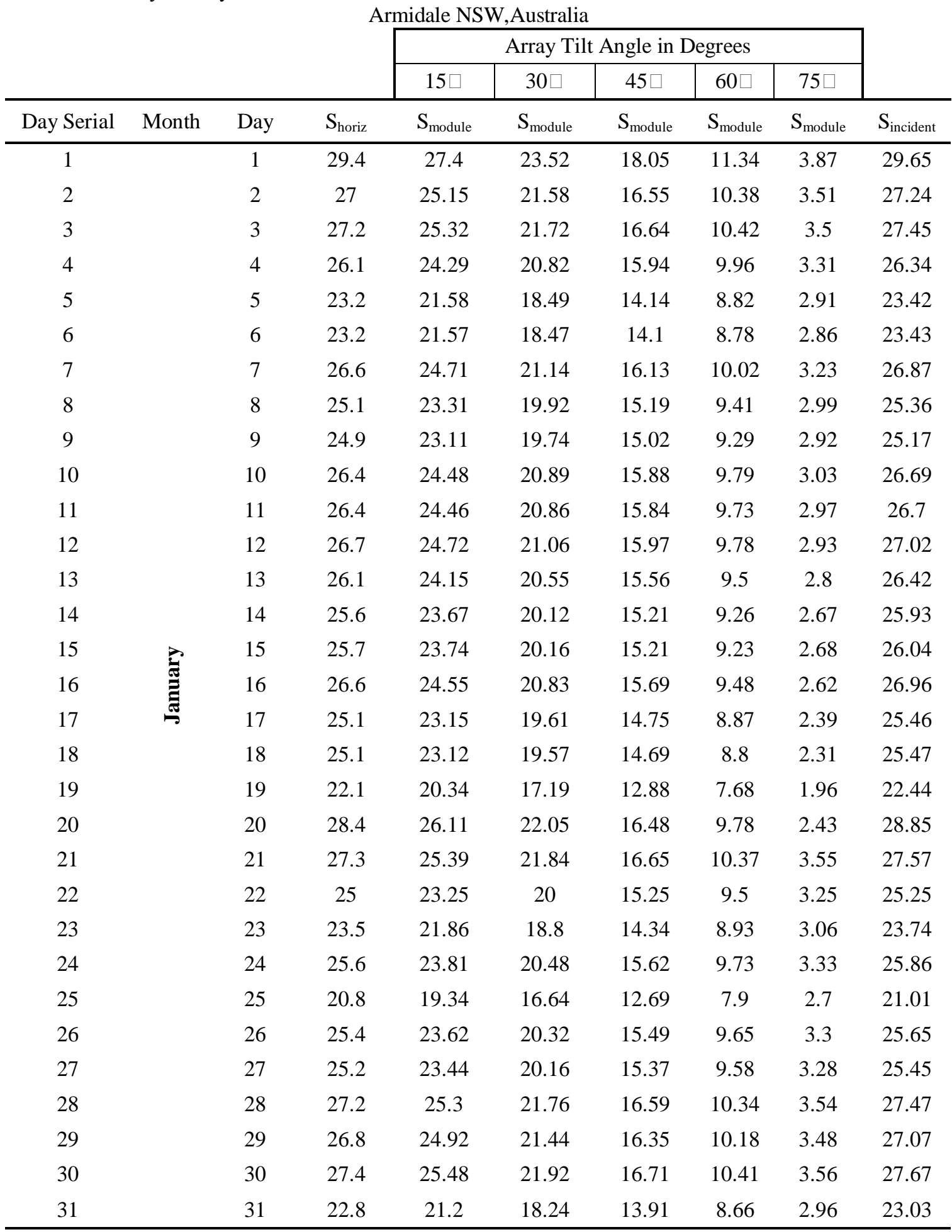


Table 5 February's Daily solar radiation on tilted surfaces valuesobtainedfromTestReferenceYear data for

\begin{tabular}{|c|c|c|c|c|c|c|c|c|c|}
\hline & \multicolumn{8}{|c|}{ Armidale NSW,Australia } & \\
\hline & & & & \multicolumn{5}{|c|}{ Array Tilt Angle in Degrees } & \\
\hline & & & & $15 \square$ & $30 \square$ & $45 \square$ & $60 \square$ & $75 \square$ & \\
\hline Day Serial & Month & Day & $\mathrm{S}_{\text {horiz }}$ & $\mathrm{S}_{\text {module }}$ & $\mathrm{S}_{\text {module }}$ & $\mathrm{S}_{\text {module }}$ & $\mathrm{S}_{\text {module }}$ & $\begin{array}{c}\mathrm{S}_{\text {modu }} \\
\text { le }\end{array}$ & $S_{\text {incident }}$ \\
\hline 32 & & 1 & 18.1 & 16.83 & 14.48 & 11.04 & 6.88 & 2.35 & 18.28 \\
\hline 33 & & 2 & 22.5 & 20.93 & 18 & 13.73 & 8.55 & 2.93 & 22.73 \\
\hline 34 & & 3 & 23.2 & 21.58 & 18.56 & 14.15 & 8.82 & 3.02 & 23.43 \\
\hline 35 & & 4 & 26.1 & 24.27 & 20.88 & 15.92 & 9.92 & 3.39 & 26.36 \\
\hline 36 & & 5 & 25.5 & 23.72 & 20.4 & 15.56 & 9.69 & 3.32 & 25.76 \\
\hline 37 & & 6 & 23.5 & 21.86 & 18.8 & 14.34 & 8.93 & 3.06 & 23.74 \\
\hline 38 & & 7 & 21.5 & 20 & 17.2 & 13.12 & 8.17 & 2.8 & 21.72 \\
\hline 39 & & 8 & 23.2 & 21.58 & 18.56 & 14.15 & 8.82 & 3.02 & 23.43 \\
\hline 40 & & 9 & 23.2 & 21.58 & 18.56 & 14.15 & 8.82 & 3.02 & 23.43 \\
\hline 41 & & 10 & 24.1 & 22.41 & 19.28 & 14.7 & 9.16 & 3.13 & 24.34 \\
\hline 42 & & 11 & 24.3 & 22.6 & 19.44 & 14.82 & 9.23 & 3.16 & 24.54 \\
\hline 43 & & 12 & 21.4 & 19.9 & 17.12 & 13.05 & 8.13 & 2.78 & 21.61 \\
\hline 44 & & 13 & 22.4 & 20.83 & 17.92 & 13.66 & 8.51 & 2.91 & 22.62 \\
\hline 45 & $\vec{Z}$ & 14 & 20.2 & 18.79 & 16.16 & 12.32 & 7.68 & 2.63 & 20.4 \\
\hline 46 & : & 15 & 19.7 & 18.32 & 15.76 & 12.02 & 7.49 & 2.56 & 19.9 \\
\hline 47 & 包 & 16 & 20.3 & 18.88 & 16.24 & 12.38 & 7.71 & 2.64 & 20.5 \\
\hline 48 & & 17 & 23.9 & 22.23 & 19.12 & 14.58 & 9.08 & 3.11 & 24.14 \\
\hline 49 & & 18 & 22.2 & 20.65 & 17.76 & 13.54 & 8.44 & 2.89 & 22.42 \\
\hline 50 & & 19 & 21.8 & 20.27 & 17.44 & 13.3 & 8.28 & 2.83 & 22.02 \\
\hline 51 & & 20 & 22.8 & 21.2 & 18.24 & 13.91 & 8.66 & 2.96 & 23.03 \\
\hline 52 & & 21 & 23.4 & 21.76 & 18.72 & 14.27 & 8.89 & 3.04 & 23.63 \\
\hline 53 & & 22 & 21.3 & 19.81 & 17.04 & 12.99 & 8.09 & 2.77 & 21.51 \\
\hline 54 & & 23 & 23.2 & 21.58 & 18.56 & 14.15 & 8.82 & 3.02 & 23.43 \\
\hline 55 & & 24 & 23.3 & 21.67 & 18.64 & 14.21 & 8.85 & 3.03 & 23.53 \\
\hline 56 & & 25 & 19 & 17.67 & 15.2 & 11.59 & 7.22 & 2.47 & 19.19 \\
\hline 57 & & 26 & 21.5 & 20 & 17.2 & 13.12 & 8.17 & 2.8 & 21.72 \\
\hline 58 & & 27 & 21.5 & 20 & 17.2 & 13.12 & 8.17 & 2.8 & 21.72 \\
\hline 59 & & 28 & 25.5 & 23.72 & 20.4 & 15.56 & 9.69 & 3.32 & 25.76 \\
\hline 60 & & 29 & 18.3 & 17.02 & 14.64 & 11.16 & 6.95 & 2.38 & 18.48 \\
\hline
\end{tabular}


Table 6 March's Daily solar radiation on tilted surfaces valuesobtainedfromTestReferenceYear data for Armidale NSW,Australia

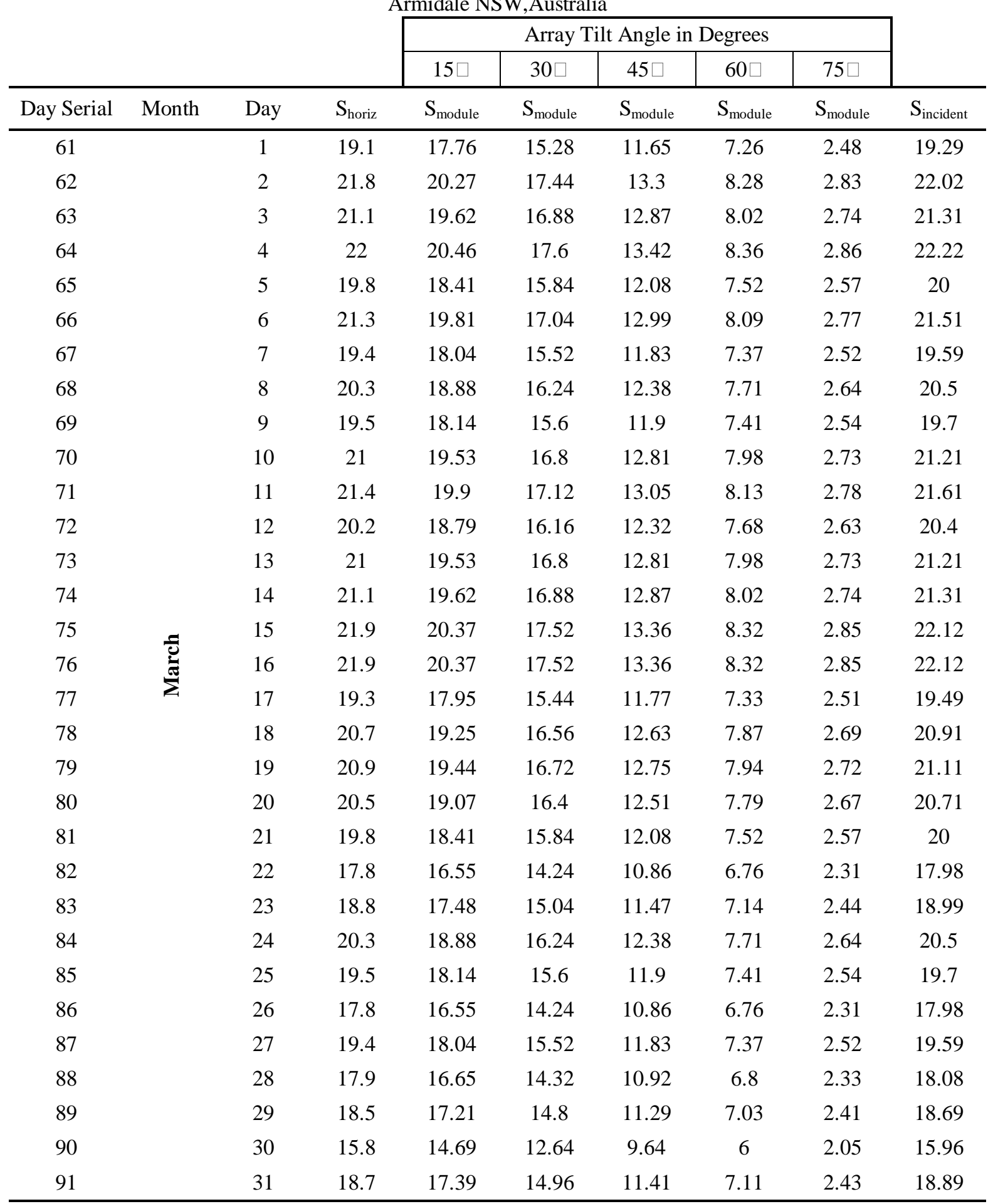


Table 7 April's Daily solar radiation on tilted surfaces valuesobtainedfromTestReferenceYear data for Armidale

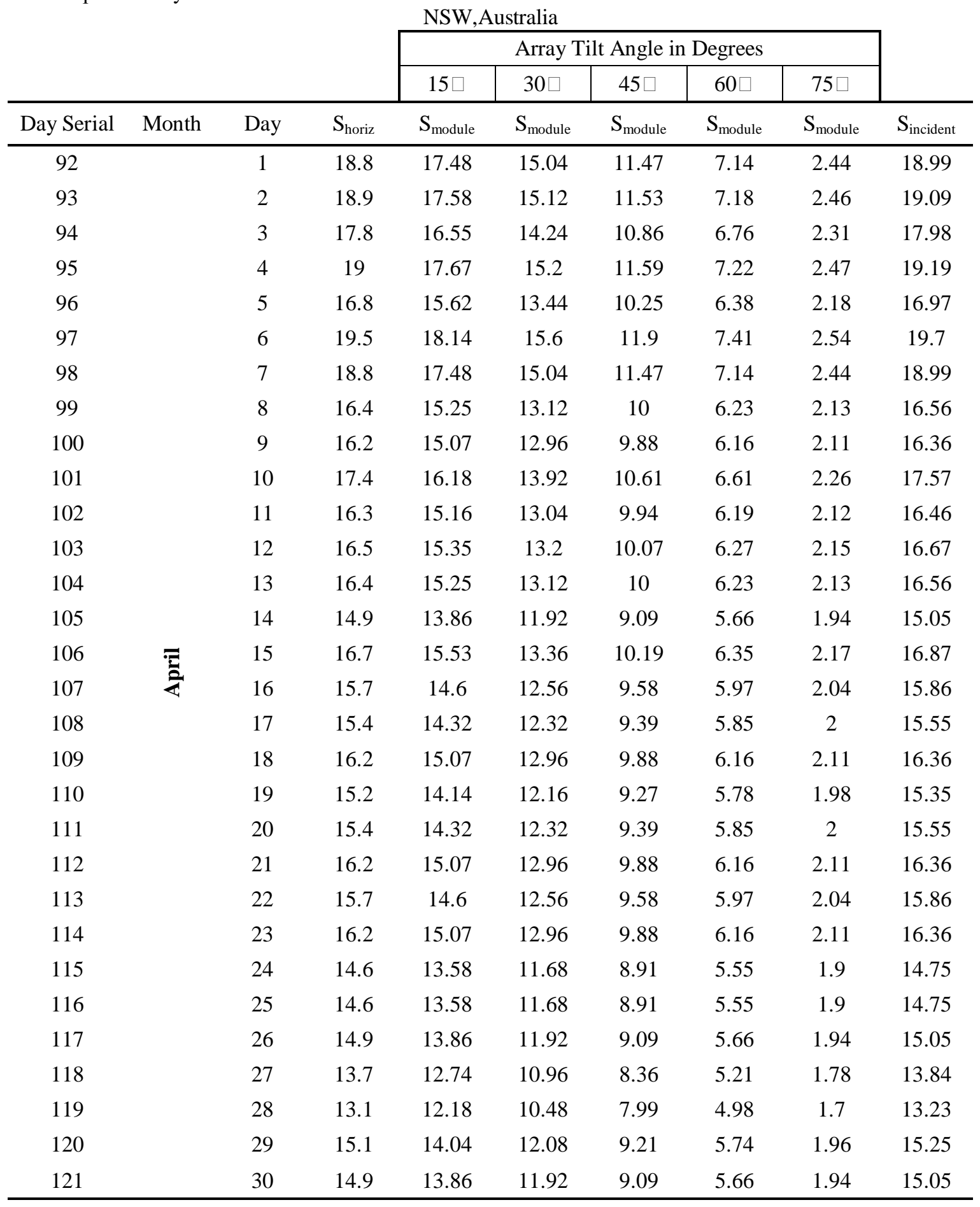


Table 8 May’s Daily solar radiation on tilted surfaces valuesobtainedfromTestReferenceYear data for Armidale

\begin{tabular}{|c|c|c|c|c|c|c|c|c|c|}
\hline & \multirow[t]{3}{*}{ ( } & \multirow[t]{3}{*}{ 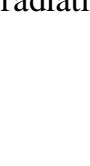 } & & \multicolumn{5}{|c|}{ NSW,Australia } & \\
\hline & & & & \multicolumn{5}{|c|}{ Array Tilt Angle in Degrees } & \\
\hline & & & & $15 \square$ & $30 \square$ & $45 \square$ & $60 \square$ & $75 \square$ & \\
\hline Day Serial & Month & Day & $\mathrm{S}_{\text {horiz }}$ & $\mathrm{S}_{\text {module }}$ & $\mathrm{S}_{\text {module }}$ & $\mathrm{S}_{\text {module }}$ & $\mathrm{S}_{\text {module }}$ & $\mathrm{S}_{\text {module }}$ & $S_{\text {incident }}$ \\
\hline 122 & & 1 & 13.5 & 12.56 & 10.8 & 8.24 & 5.13 & 1.76 & 13.64 \\
\hline 123 & & 2 & 12.6 & 11.72 & 10.08 & 7.69 & 4.79 & 1.64 & 12.73 \\
\hline 124 & & 3 & 13.9 & 12.93 & 11.12 & 8.48 & 5.28 & 1.81 & 14.04 \\
\hline 125 & & 4 & 13 & 12.09 & 10.4 & 7.93 & 4.94 & 1.69 & 13.13 \\
\hline 126 & & 5 & 12 & 11.16 & 9.6 & 7.32 & 4.56 & 1.56 & 12.12 \\
\hline 127 & & 6 & 14.7 & 13.67 & 11.76 & 8.97 & 5.59 & 1.91 & 14.85 \\
\hline 128 & & 7 & 14.5 & 13.49 & 11.6 & 8.85 & 5.51 & 1.89 & 14.65 \\
\hline 129 & & 8 & 12.8 & 11.9 & 10.24 & 7.81 & 4.86 & 1.66 & 12.93 \\
\hline 130 & & 9 & 13.4 & 12.46 & 10.72 & 8.17 & 5.09 & 1.74 & 13.53 \\
\hline 131 & & 10 & 12.9 & 12 & 10.32 & 7.87 & 4.9 & 1.68 & 13.03 \\
\hline 132 & & 11 & 12.8 & 11.9 & 10.24 & 7.81 & 4.86 & 1.66 & 12.93 \\
\hline 133 & & 12 & 12.6 & 11.72 & 10.08 & 7.69 & 4.79 & 1.64 & 12.73 \\
\hline 134 & & 13 & 12.5 & 11.63 & 10 & 7.63 & 4.75 & 1.63 & 12.63 \\
\hline 135 & & 14 & 12.7 & 11.81 & 10.16 & 7.75 & 4.83 & 1.65 & 12.83 \\
\hline 136 & & 15 & 12.9 & 12 & 10.32 & 7.87 & 4.9 & 1.68 & 13.03 \\
\hline 137 & $\vec{\pi}$ & 16 & 12.2 & 11.35 & 9.76 & 7.44 & 4.64 & 1.59 & 12.32 \\
\hline 138 & & 17 & 11.5 & 10.7 & 9.2 & 7.02 & 4.37 & 1.5 & 11.62 \\
\hline 139 & & 18 & 11.1 & 10.32 & 8.88 & 6.77 & 4.22 & 1.44 & 11.21 \\
\hline 140 & & 19 & 12.4 & 11.53 & 9.92 & 7.56 & 4.71 & 1.61 & 12.52 \\
\hline 141 & & 20 & 12.4 & 11.53 & 9.92 & 7.56 & 4.71 & 1.61 & 12.52 \\
\hline 142 & & 21 & 11.3 & 10.51 & 9.04 & 6.89 & 4.29 & 1.47 & 11.41 \\
\hline 143 & & 22 & 11.9 & 11.07 & 9.52 & 7.26 & 4.52 & 1.55 & 12.02 \\
\hline 144 & & 23 & 12.2 & 11.35 & 9.76 & 7.44 & 4.64 & 1.59 & 12.32 \\
\hline 145 & & 24 & 12 & 11.16 & 9.6 & 7.32 & 4.56 & 1.56 & 12.12 \\
\hline 146 & & 25 & 12.2 & 11.35 & 9.76 & 7.44 & 4.64 & 1.59 & 12.32 \\
\hline 147 & & 26 & 11.9 & 11.07 & 9.52 & 7.26 & 4.52 & 1.55 & 12.02 \\
\hline 148 & & 27 & 12.2 & 11.35 & 9.76 & 7.44 & 4.64 & 1.59 & 12.32 \\
\hline 149 & & 28 & 11.7 & 10.88 & 9.36 & 7.14 & 4.45 & 1.52 & 11.82 \\
\hline 150 & & 29 & 11.4 & 10.6 & 9.12 & 6.95 & 4.33 & 1.48 & 11.51 \\
\hline 151 & & 30 & 11.5 & 10.7 & 9.2 & 7.02 & 4.37 & 1.5 & 11.62 \\
\hline 152 & & 31 & 11 & 10.23 & 8.8 & 6.71 & 4.18 & 1.43 & 11.11 \\
\hline
\end{tabular}


Table 9 June's Daily solar radiation on tilted surfaces valuesobtainedfromTestReferenceYear data for Armidale

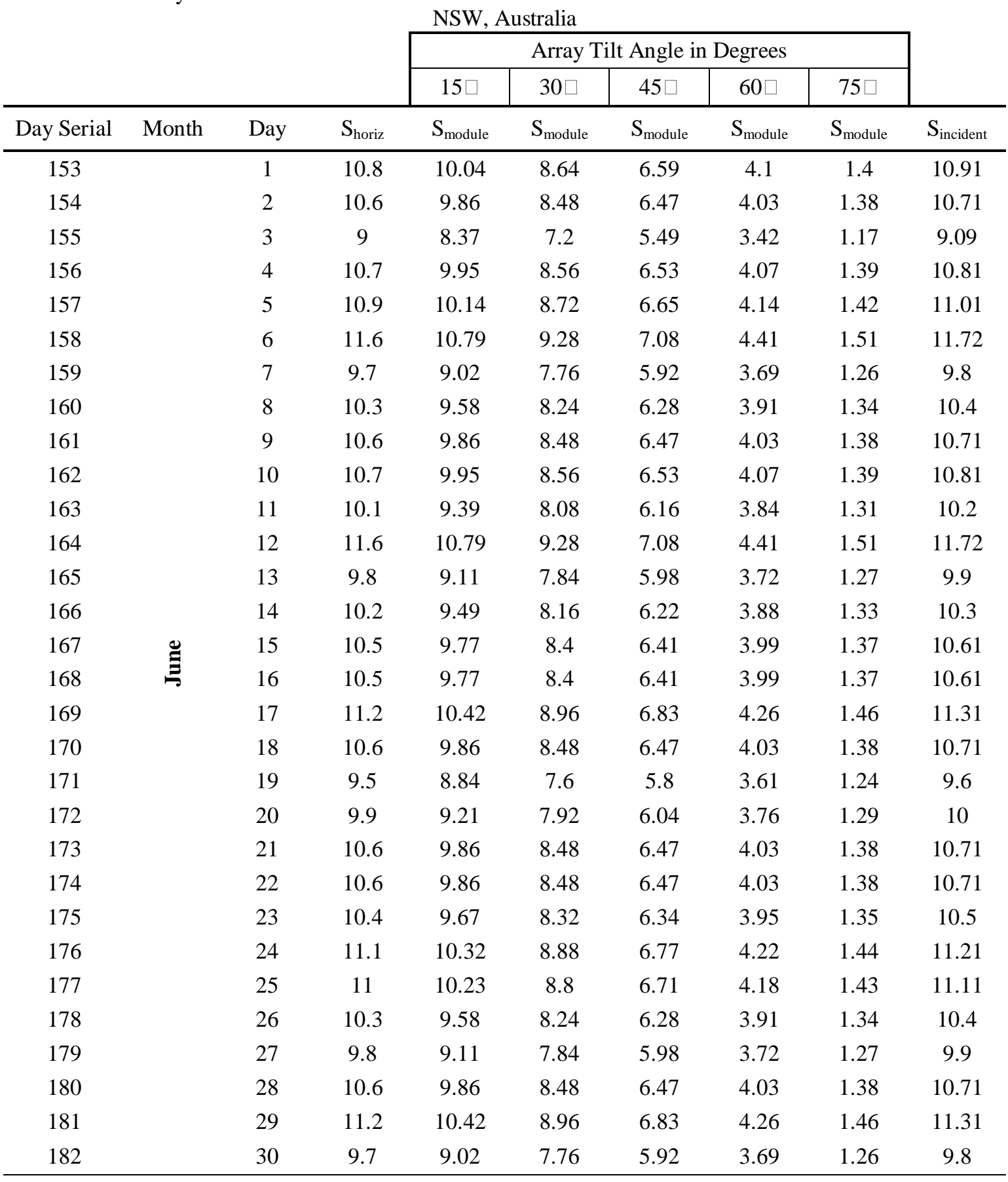


Table 10 July's Daily solar radiation on tilted surfaces valuesobtainedfromTestReferenceYear data for Armidale

\begin{tabular}{|c|c|c|c|c|c|c|c|c|c|}
\hline & \multicolumn{5}{|c|}{ NSW, Australia } & \\
\hline & & & & \multicolumn{5}{|c|}{ Array Tilt Angle in Degrees } & \\
\hline & & & & $15 \square$ & $30 \square$ & $45 \square$ & $60 \square$ & $75 \square$ & \\
\hline Day Serial & Month & Day & $S_{\text {horiz }}$ & $\mathrm{S}_{\text {module }}$ & $\mathrm{S}_{\text {module }}$ & $\mathrm{S}_{\text {module }}$ & $\mathrm{S}_{\text {module }}$ & $\mathrm{S}_{\text {module }}$ & $\mathrm{S}_{\text {incident }}$ \\
\hline 183 & & 1 & 10 & 9.3 & 8 & 6.1 & 3.8 & 1.3 & 10.1 \\
\hline 184 & & 2 & 10.4 & 9.67 & 8.32 & 6.34 & 3.95 & 1.35 & 10.5 \\
\hline 185 & & 3 & 11.2 & 10.42 & 8.96 & 6.83 & 4.26 & 1.46 & 11.31 \\
\hline 186 & & 4 & 10 & 9.3 & 8 & 6.1 & 3.8 & 1.3 & 10.1 \\
\hline 187 & & 5 & 12 & 11.16 & 9.6 & 7.32 & 4.56 & 1.56 & 12.12 \\
\hline 188 & & 6 & 11.2 & 10.42 & 8.96 & 6.83 & 4.26 & 1.46 & 11.31 \\
\hline 189 & & 7 & 10.8 & 10.04 & 8.64 & 6.59 & 4.1 & 1.4 & 10.91 \\
\hline 190 & & 8 & 10.7 & 9.95 & 8.56 & 6.53 & 4.07 & 1.39 & 10.81 \\
\hline 191 & & 9 & 9.6 & 8.93 & 7.68 & 5.86 & 3.65 & 1.25 & 9.7 \\
\hline 192 & & 10 & 11.2 & 10.42 & 8.96 & 6.83 & 4.26 & 1.46 & 11.31 \\
\hline 193 & & 11 & 11.4 & 10.6 & 9.12 & 6.95 & 4.33 & 1.48 & 11.51 \\
\hline 194 & & 12 & 10.3 & 9.58 & 8.24 & 6.28 & 3.91 & 1.34 & 10.4 \\
\hline 195 & & 13 & 9.9 & 9.21 & 7.92 & 6.04 & 3.76 & 1.29 & 10 \\
\hline 196 & & 14 & 10.2 & 9.49 & 8.16 & 6.22 & 3.88 & 1.33 & 10.3 \\
\hline 197 & & 15 & 11.6 & 10.79 & 9.28 & 7.08 & 4.41 & 1.51 & 11.72 \\
\hline 198 & $\vec{\Xi}$ & 16 & 12.4 & 11.53 & 9.92 & 7.56 & 4.71 & 1.61 & 12.52 \\
\hline 199 & & 17 & 12.1 & 11.25 & 9.68 & 7.38 & 4.6 & 1.57 & 12.22 \\
\hline 200 & & 18 & 10.7 & 9.95 & 8.56 & 6.53 & 4.07 & 1.39 & 10.81 \\
\hline 201 & & 19 & 12.2 & 11.35 & 9.76 & 7.44 & 4.64 & 1.59 & 12.32 \\
\hline 202 & & 20 & 12.4 & 11.53 & 9.92 & 7.56 & 4.71 & 1.61 & 12.52 \\
\hline 203 & & 21 & 12.4 & 11.53 & 9.92 & 7.56 & 4.71 & 1.61 & 12.52 \\
\hline 204 & & 22 & 12.2 & 11.35 & 9.76 & 7.44 & 4.64 & 1.59 & 12.32 \\
\hline 205 & & 23 & 12.7 & 11.81 & 10.16 & 7.75 & 4.83 & 1.65 & 12.83 \\
\hline 206 & & 24 & 11.6 & 10.79 & 9.28 & 7.08 & 4.41 & 1.51 & 11.72 \\
\hline 207 & & 25 & 10.8 & 10.04 & 8.64 & 6.59 & 4.1 & 1.4 & 10.91 \\
\hline 208 & & 26 & 9.6 & 8.93 & 7.68 & 5.86 & 3.65 & 1.25 & 9.7 \\
\hline 209 & & 27 & 11.4 & 10.6 & 9.12 & 6.95 & 4.33 & 1.48 & 11.51 \\
\hline 210 & & 28 & 11.7 & 10.88 & 9.36 & 7.14 & 4.45 & 1.52 & 11.82 \\
\hline 211 & & 29 & 13.6 & 12.65 & 10.88 & 8.3 & 5.17 & 1.77 & 13.74 \\
\hline 212 & & 30 & 13.3 & 12.37 & 10.64 & 8.11 & 5.05 & 1.73 & 13.43 \\
\hline 213 & & 31 & 12.8 & 11.9 & 10.24 & 7.81 & 4.86 & 1.66 & 12.93 \\
\hline
\end{tabular}


Table 11 August's Daily solar radiation on tilted surfaces valuesobtainedfromTestReferenceYear data for

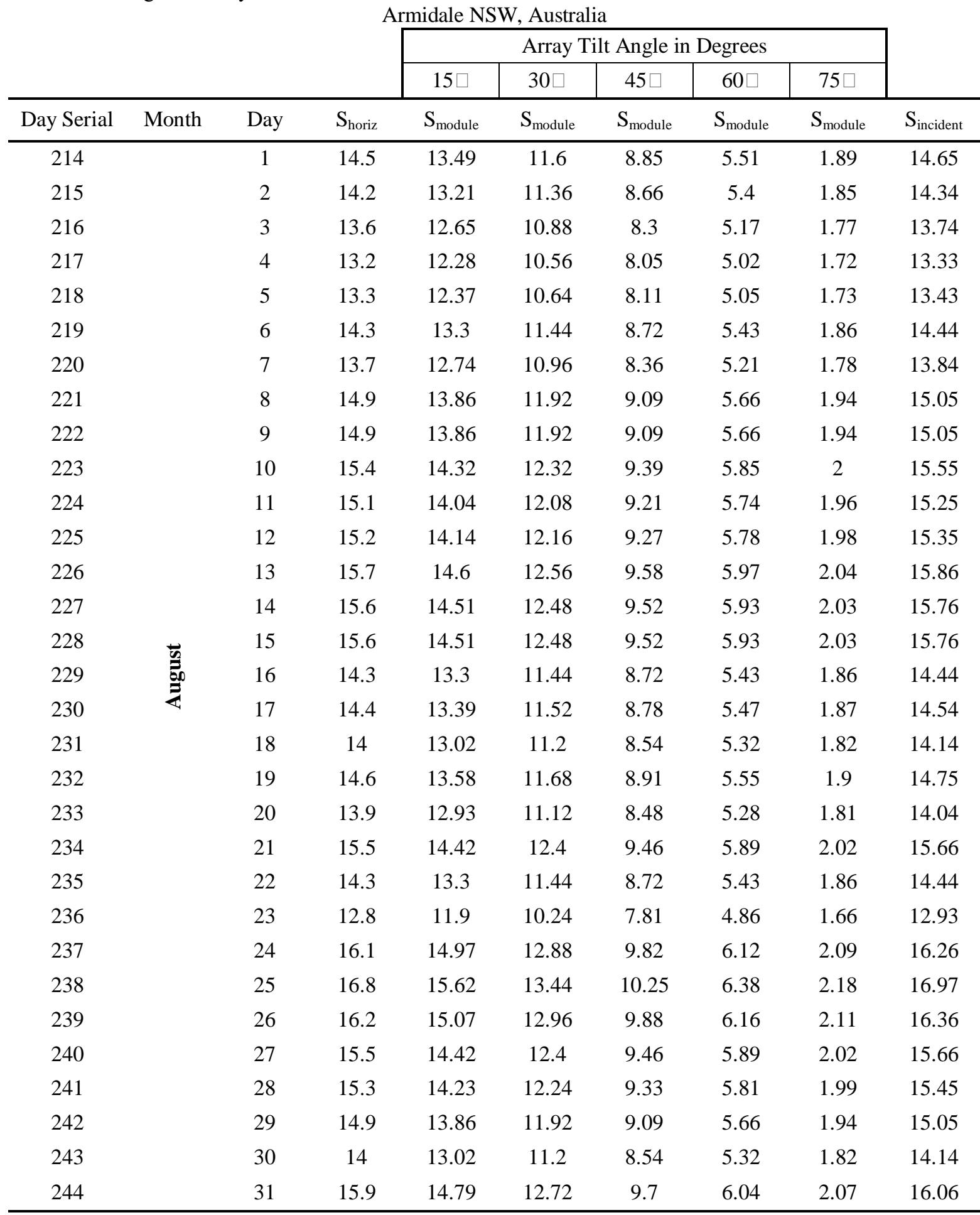


Table 12 September's Daily solar radiation on tilted surfaces valuesobtainedfromTestReferenceYear data for

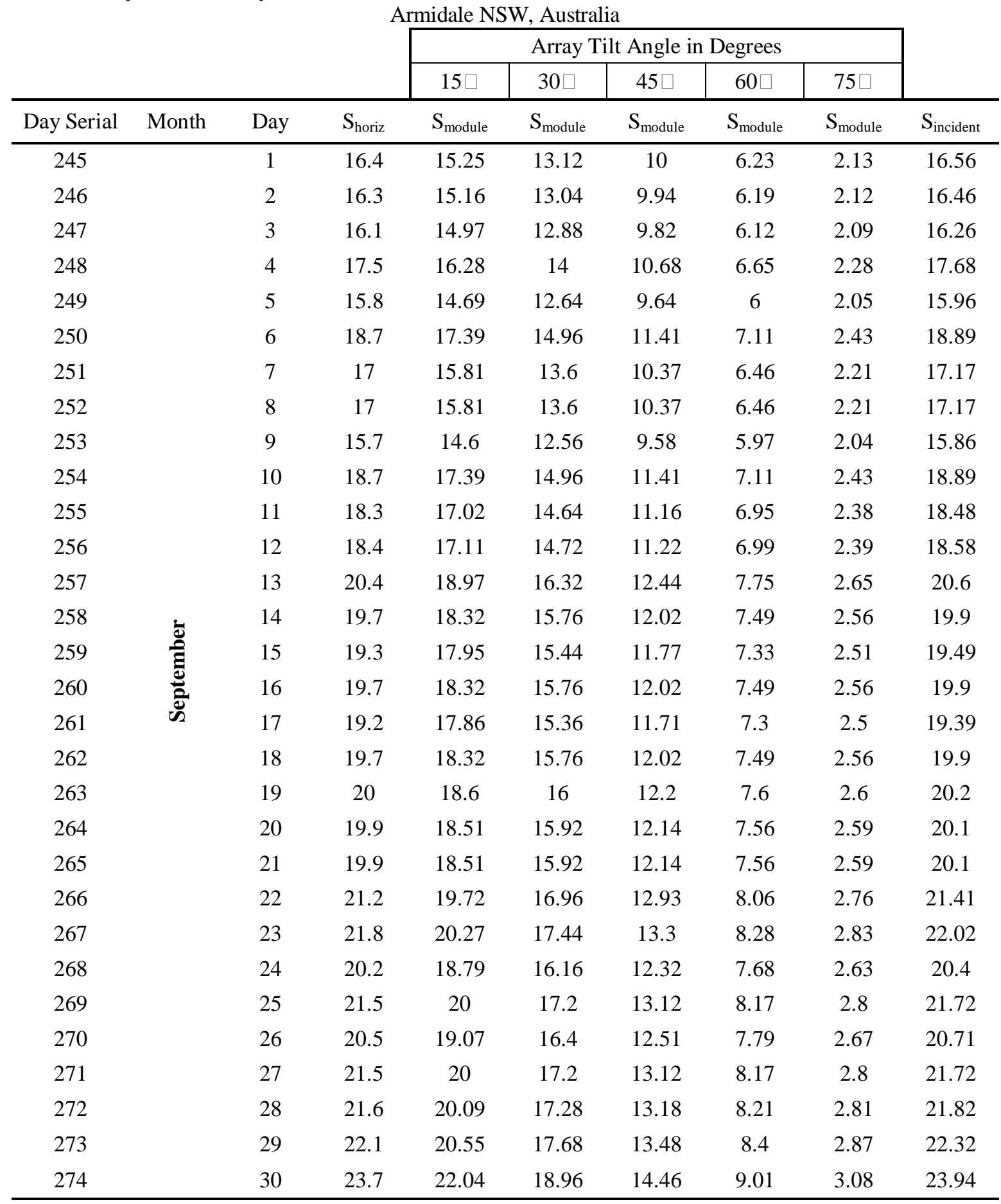


Table 13 October's Daily solar radiation on tilted surfaces valuesobtainedfromTestReferenceYear data for

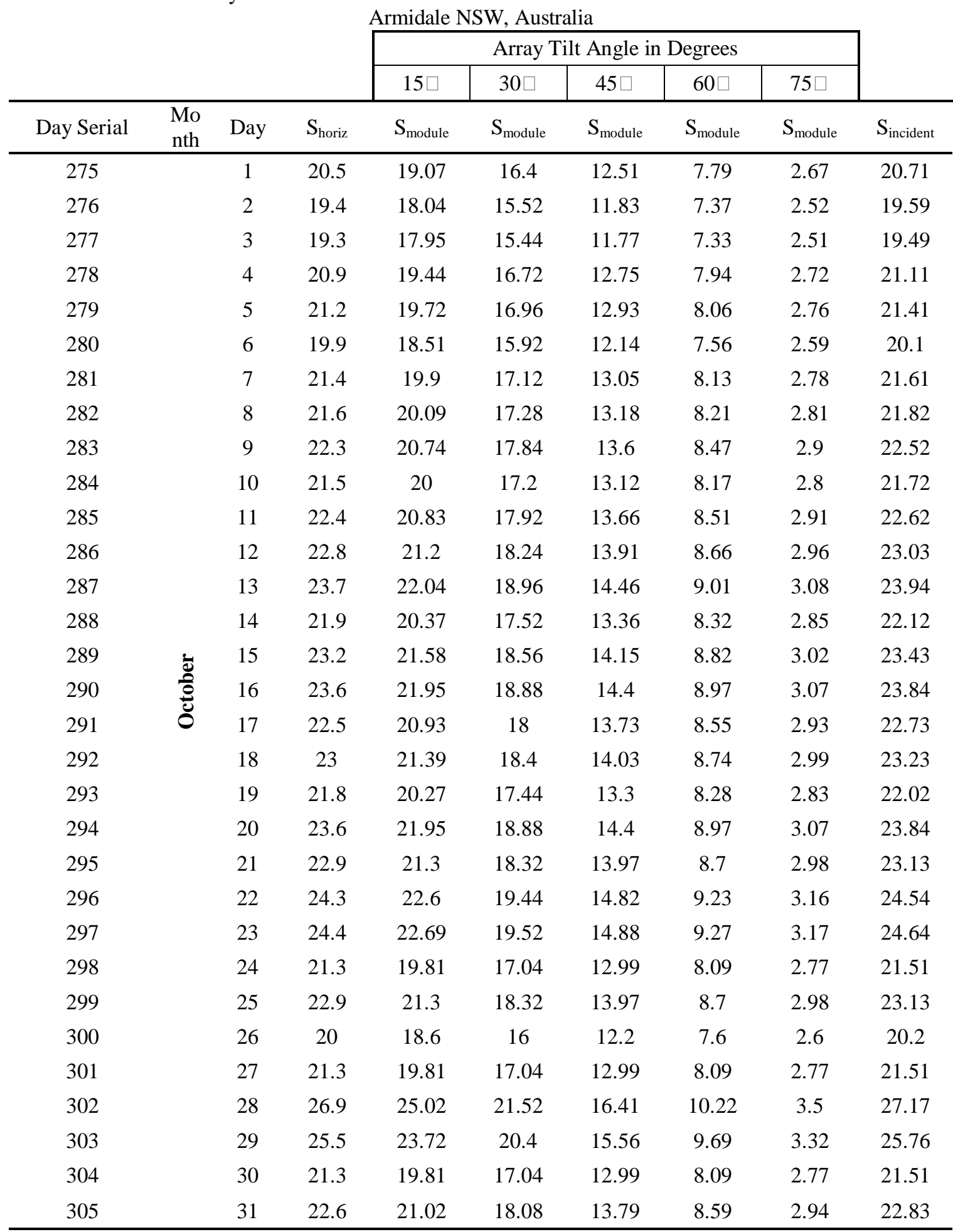


Table 14 November's Daily solar radiation on tilted surfaces valuesobtainedfromTestReferenceYear data for

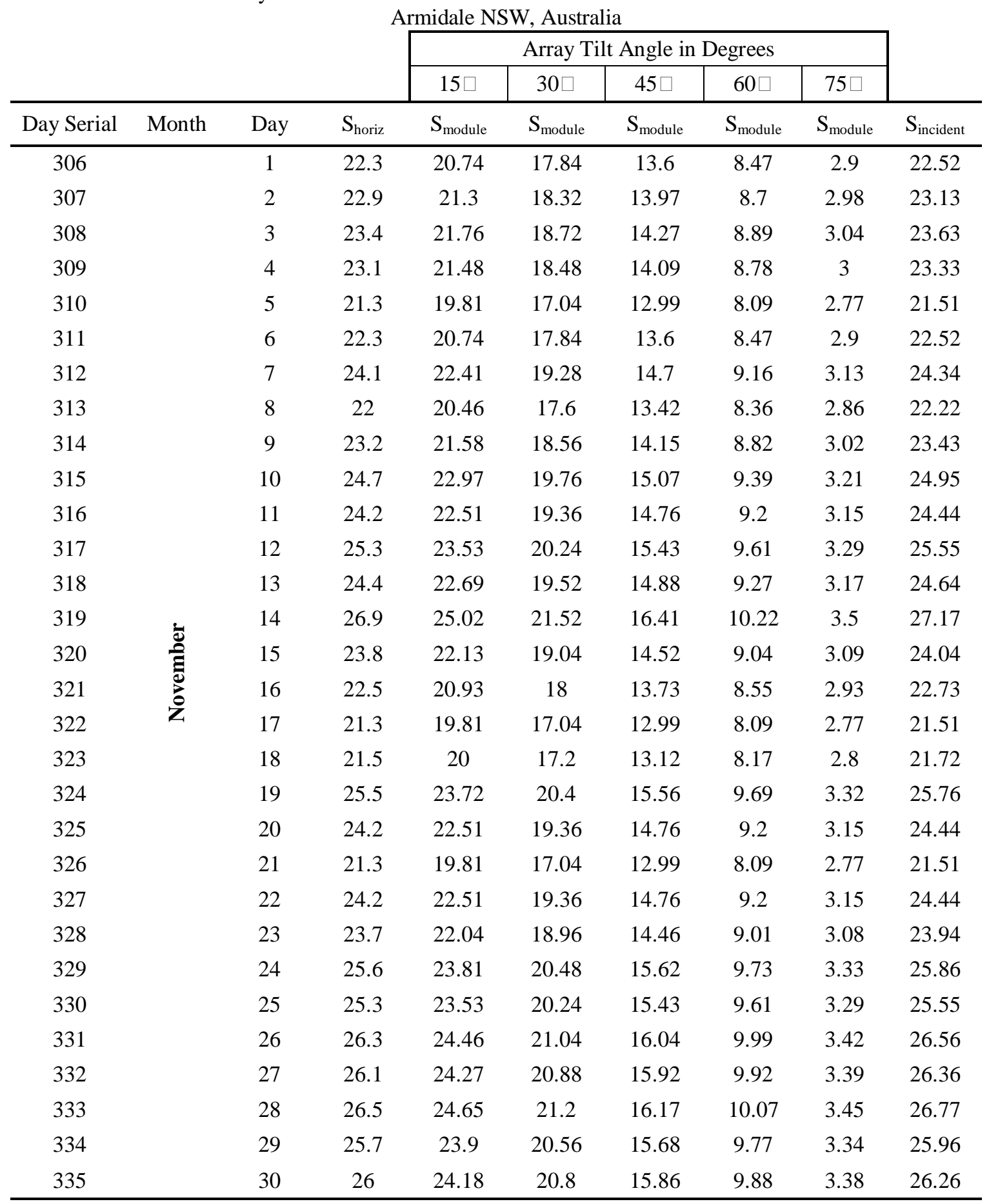


Table 15 December's Daily solar radiation on tilted surfaces valuesobtainedfromTestReferenceYear data for

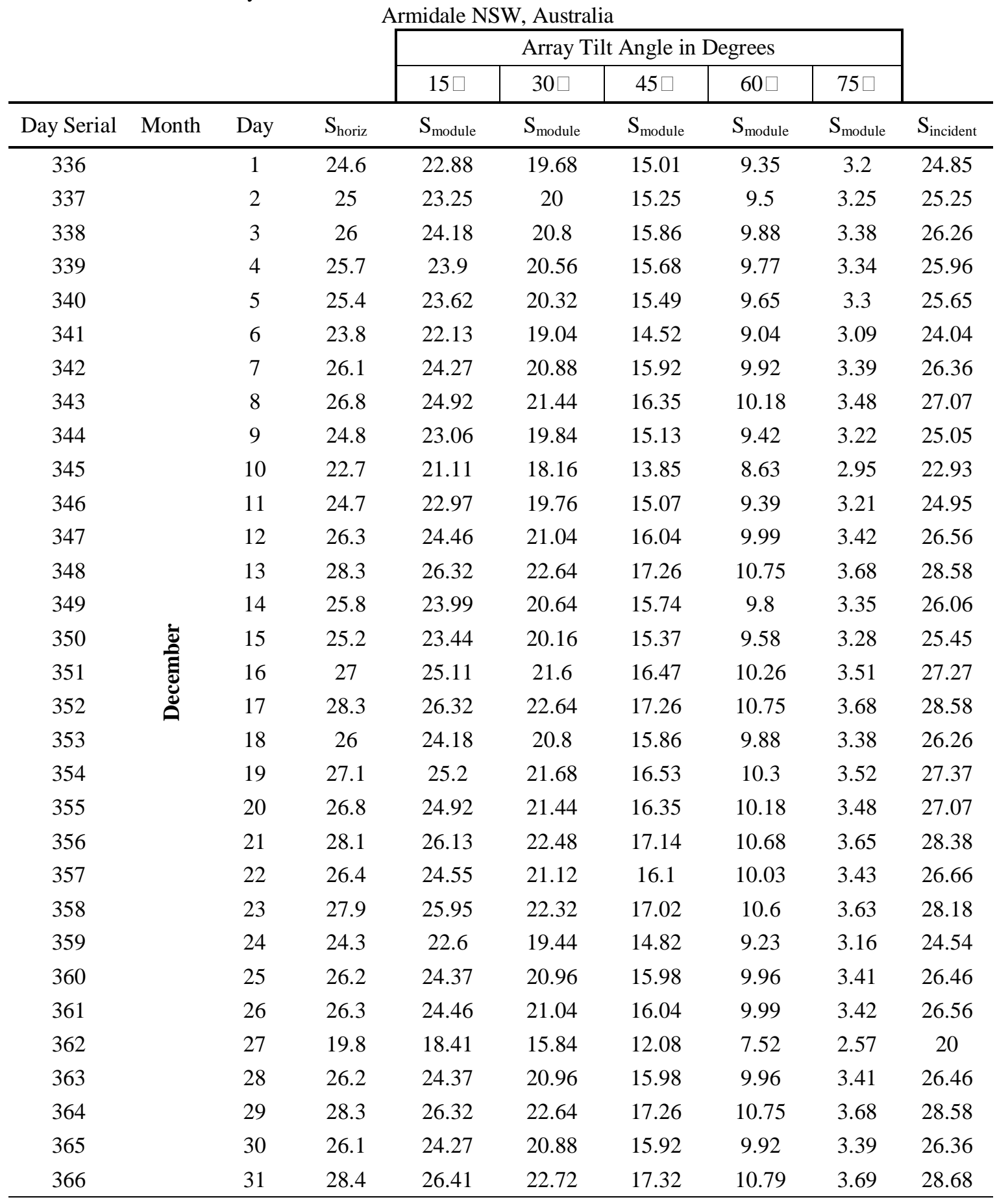

\section{V.}

\section{CONCLUSION}

The globalsolar radiation onhorizontalsurfaceshas beencollected and calculations have been conducted tocalculatethemonthly averagedailysolarirradianceoninclinedsurfacesfromtheglobalsolarirradiance.

Typical solar radiation data is very important for calculations concerning many solar energy generation systems and for building energy calculation modelling and analysis. In this study, test reference years for daily solar radiationon tilted surfaces with prescribed angles $\left(15^{\circ}, 30^{\circ}, 45^{\circ}, 60^{\circ}\right.$ and $\left.75^{\circ}\right)$ for Armidale town NSW, Australia are generated using 23 years of the meteorologically measured data. As well, the results show that Armidale has a high potential of solar energy through most of the year except for the winter months, which is still potential but not high. The generated test reference year would be a very useful reference for solar energy systems designers for any type of building applications as it helps calculating the actual energy generated during the daily sun movements around fixed solar arrays. 
It is worth mentioning that well such TYM study has been developed for Armidale for mean wind speed measured at 10 meter height [19]. So concurrently with this study, hybrid solar and wind energy systems can be investigated, examined and designed for Armidale based on this study and study [16] concurrently.

\section{REFERENCES}

[1] A. Argiriou, S. Lykoudis, S. Kontoyiannidis, C.A. Balaras, D. Asimakopoulos, M. Petrakis, and P.Kassomenos. Comparison of methodologies for TMY generation using 20 years data forAthens, Greece. Solar Energy 66(1), 1999, 33-45.

[2] W. Marion and K. Urban. User's Manual for TMY2s. National Renewable Energy Laboratory, Colorado, USA, 1995.

[3] H. Bulut. Generation of typical solar radiation data for Istanbul, Turkey. International Journal of Energy Research 27(9), 2003, 847-855.

[4] H. Bulut. Typical Solar Radiation Year for South-eastern Anatolia. Renewable Energy 29(9), 2004, $1477-1488$.

[5] R.L. Fagbenle. Generation of a test reference year for Ibadan, Nigeria. Energy Conversion andManagement 30(1), 1995, 61-63.

[6] J.C. Lam, S.C.M. Hui, and A.L.S. Chan. A statistical approach to the development of a typicalmeteorological year for Hong Kong. Architectural Science Review 39(4), 1996, 201-209.

[7] A. Miguel, and J. Bilbao. Test reference year generation from meteorological and simulatedsolar radiation data. Solar Energy 78(6), 2005, 695-703.

[8] M. Petrakis, H.D. Kambezidis, S. Lykoudis, A.D. Adamopoulos, P. Kassomenos, I.M. Michaelides, S.A. Kalogirou, G. Roditis, I. Chrysis, and A. Hadjigianni. Generation of a typical meteorological year for Nicosia, Cyprus. Renewable Energy: 13(3), 1998, 381-388.

[9] S.A.M. Said and H.M. Kadry. Generation of representative weather-year data for SaudiArabia. Applied Energy 48(2), 1994, 131-136.

[10] M.A.M. Shaltout and M.T.Y. Tadros. Typical solar radiation year for Egypt. Renewable Energy 4(4), 1994, 387-393.

[11] J.M. Finkelstein and R.E. Schafer. Improved goodness of fit tests. Biometrika 58(3), 1971, 641-645.

[12] G. Kalogirou, I.Roditis, II. Chrysis, and A. Hadjigianni. Generation of a typical meteorological year for Nicosia, Cyprus. Renewable Energy: 13(3), 1998, 381-388.

[13] L. Q. Liu and Z. X. Wang. The development and application practice of wind-solar energy hybrid generation systems in China.Renewable and Sustainable Energy Review 13(6-7), 2009, 1504-1512.

[14] T.N Anderson, M. Duke and J.K. Carson. Generationof a typical meteorological year for Harcourt zone. Journal of Engineering Science and Technology 6(2), 2011, 204-2014

[15] Y. Maklad. Generation of an Annual Typical Meteorological Solar Radiation for Armidale NSW, Australia. IOSR Journal of Engineering (IOSRJEN) 4(4), 2014, 41-45.

[16] J.E. Hay and D.C. McKay. Estimating solar irradiance on inclined surfaces: a review and assessment of methodologies. International Journal of Sustainable Energy, 3 (4-5), 1985, 203-240.

[17] S.S. Chandel, R.K. AggarwalandA.N. Pandey. New correlation to estimate global solar radiation on horizontal surfaces using sunshine hour and temperature data for Indian sites. Solar Energy Engineering Journal, 127 (3), 2005, 417-420.

[18] C. Gueymard. Prediction and performance assessment of mean hourly global radiation. Solar Energy, 68 (3), 2000, 285-303.

[19] Y. Maklad. Generation of an Annual Typical Meteorological Wind Speed for Armidale NSW, Australia. IOSR Journal of Engineering (IOSRJEN) 4(7), 2014. 